\title{
The small DdrR protein directly interacts with the UmuDAb regulator to inhibit the mutagenic DNA damage response in Acinetobacter baumannii
}

\author{
Anja Pavlin ${ }^{1}$, Gregor Bajc ${ }^{1}$, Nadine Fornelos ${ }^{2}$, Douglas F. Browning ${ }^{3}$, Matej Butala ${ }^{1 *}$ \\ ${ }^{1}$ Department of Biology, Biotechnical Faculty, University of Ljubljana, Ljubljana 1000, \\ Slovenia \\ ${ }^{2}$ Broad Institute of MIT and Harvard, Cambridge, Massachusetts 02142, USA \\ ${ }^{3}$ Institute of Microbiology and Infection, University of Birmingham, Birmingham, B15 2TT, \\ UK
}

Corresponding author: Matej Butala

Address: Department of Biology, Biotechnical Faculty, University of Ljubljana, Večna pot 111, Ljubljana, Slovenia

Phone: +38613203397

Fax: +38612573390

Email: matej.butala@bf.uni-lj.si

\section{Disclaimer statement:}

The authors have withdrawn their manuscript due to observed inconsistent results regarding the DdrR effect on the enhanced binding of UmuDAb to its promoter regions. Further experiments are needed to unravel the biology of DdrR. Therefore, the authors do not wish this work to be cited as a reference for the project. If you have any questions, please contact the corresponding author. 